\title{
Logistic Equation Revisited: The Two-Sex case
}

\author{
Carlos Castillo-Chavez \\ Biometrics Unit and \\ Center for Applied Mathematics \\ Cornell University \\ Ithaca, NY 14853
}

\author{
Wenzhang Huang \\ Biometrics Unit and \\ Center for Applied Mathematics \\ Cornell University \\ Ithaca, NY 14853
}




\title{
The Logistic Equation Revisited: The Two-Sex case
}

\author{
Carlos Castillo-Chavez and Wenzhang Huang \\ Biometrics Unit/Center for Applied Mathematics \\ Cornell University \\ May, 1994
}

Dedicated to the memory of Stavros Busenberg

\begin{abstract}
The goal of this article is to formulate and analyze the simplest logistic pair-formation model and to contrast its dynamics to that of the corresponding Malthusian pair-formation model, that is, the Kendall/Keyfitz and Dietz/Hadeler model. The Malthusian pairformation model supports a unique nontrivial stable exponential solution and we show that the logistic pair-formation model also supports a unique stable nontrivial bounded solution.
\end{abstract}

\section{Introduction}

The simplest mathematical model in demography is known as Malthus' Law. If the population of individuals at time $t$ is denoted by $P(t)$, then Malthus Law is given by the solution to the simple differential equation

$$
\frac{d P}{d t}=\beta P, \quad P(0)=P_{0}
$$

where $\beta$ denotes the per capita growth rate of the population. This model was introduced in 1789 in one of the most influential papers in demography and population dynamics. 
The model assumes a constant per capita growth rate which leads either to extinction or to population explosion (unless $\beta=0$ ). The acknowledgement of existence of finite resources (the carrying capacity of an ecosystem) requires the introduction of models that cannot support exponential growth indefinitely. The assumption of a per-capita growth rate $\beta$ that depends on the size of the population led theoreticians to study the model

$$
\frac{d P}{d t}=\beta(P) P, \quad P(0)=P_{0},
$$

where it was assumed that

$$
\beta(P) \geq 0, \quad \frac{d \beta(P)}{d P} \leq 0 .
$$

The most common example is provided by the logistic equation (introduced by Verhulst $1845,1847)$ in which

$$
\beta(P)=r\left(1-\frac{P}{K}\right),
$$

and which led biologists to the theory of $r-K$ selection (see for example May 1974).

These models have played a very useful role in theoretical biology (see May 1974). However, because their use is restricted to modeling the dynamics of a homogeneous singlesex population, these models and their single-sex generalizations, such as Leslie's model (Leslie 1945), cannot take into account gender-related factors central to the study of the life history of real populations.

The use of two-sex models in demography was introduced by Kendall (1949) and Keyfitz (1949) and was further developed by Fredrickson (1971), Pollard (1973), and McFarland (1972). The central problem associated with the formulation of two-sex demographic models is that of modeling the nonlinear process of pairing. This modeling problem has been the object of intense research over the last few years. A recent overview of this problems and some of the current solutions can be found in Castillo-Chavez and Busenberg (1991), Castillo-Chavez et al. (1994a, b).

Following the recent work of Dietz and Hadeler (1988), we will denote the rate of pairformation by $\phi$. The work of Fredrickson, MacFarland, Keyfitz, Pollard, and Kendall (cited above) proposes various functional forms of $\phi$. All these forms had as independent variables the state variables that describe the population sizes of single males and single females. From some of these examples that model the nonlinear process of pairing, Fredrickson (1971) and others (see Hoppensteadt 1974 and references therein) extracted a set of basic properties that must be satisfied by the rate of pair formation $\phi$. They include

$$
\begin{gathered}
\phi(m, f) \geq 0 \\
\phi(m+u, f+v) \geq \phi(m, f) \\
\phi(\alpha m, \alpha f)=\alpha \phi(m, f), \\
\phi(m, 0)=\phi(0, f)=0,
\end{gathered}
$$


where $m$ denotes the population of single males, $f$ the population of single females, and $m, f, u, v$ and the constant $\alpha$ are assumed to be nonnegative. Fredrickson and others observed that some of the pairing functions such as the harmonic mean satisfy these properties while others did not. In 1988, Dietz and Hadeler analyzed their generalized version of the Kendall-Keyfitz pair-formation model. Their analysis was further extended by Waldstätter (1989). The Dietz/Hadeler model provides the simplest two-sex demographic model with general pair-formation rates. The model is nonlinear; however, it is homogeneous of order one and hence it supports exponential solutions. Consequently, the Dietz/Hadeler model can be thought of as the natural generalization of the Malthus model to two-sex populations. Obviously not all populations exhibit (at least on relatively long time scales) exponential growth and therefore we can argue as did Verhulst $(1845,1847)$ or Gurtin and MacCamy (1974) that birth (and separation) rates are nonlinear functions of the number of paired individuals.

The focus of this paper is to formulate and analyze the simplest logistic pair-formation model and to contrast its dynamics to that of the corresponding Malthusian pair-formation model. The Malthusian model supports a unique nontrivial stable exponential solution and we will show that the logistic pair-formation model also supports a unique stable nontrivial bounded solution. We will further specify the precise demographic conditions on which this behavior takes place. A description of our results requires a brief but complete summary of the results associated with the Malthusian pair-formation model, which is given in Section 2. Section 3 formulates the simplest logistic pair-formation model and states and interprets the results of our analysis. Section 4 collects the proofs associated with the analysis of the logistic pair-formation model, while Section 5 collects our final thoughts and outlines future research.

\section{The Dietz/Hadeler Model}

We begin by formulating Dietz/Hadeler's model as well as providing a summary of their results. We let $m_{s}(t)$ denote the population of single males at time $t, f_{s}(t)$ the population of single females at time $t$, and $p(t)$ the population of paired individuals (heterosexual pairing) at time $t$. Furthermore, we let $\mu_{m}$ and $\mu_{f}$ denote the per-capita death rates for males and females, and $\beta_{m}, \beta_{f}$ the per-capita birth rates for males and females while $\sigma$ denotes the per-pair separation rate. Using these definitions we arrive at the following demographic pair-formation model:

$$
\begin{aligned}
\dot{m}_{s} & =-\mu_{m} m_{s}+\left(\beta_{m}+\mu_{f}+\sigma\right) p-\phi\left(m_{s}, f_{s}\right) \\
\dot{f}_{s} & =-\mu_{f} f_{s}+\left(\beta_{f}+\mu_{m}+\sigma\right) p-\phi\left(m_{s}, f_{s}\right) \\
\dot{p} & =-\left(\mu_{m}+\mu_{f}+\sigma\right) p+\phi\left(m_{s}, f_{s}\right),
\end{aligned}
$$


where $\phi$ denotes the pair-formation rate. It is assumed that $\phi$ is differentiable for $\left(m_{s}, f_{s}\right) \in$ $\mathbb{R}_{+}^{2} \backslash\{(0,0)\}$ and it is assumed to satisfy properties (PF). We observe that system (2.1) is a homogeneous system of degree one and, consequently, it supports exponential solutions. Hadeler and collaborators have developed an extensive theory for homogeneous systems of this type. The following results found in Dietz \& Hadeler (1988) or Waldstätter (1989), make use of this theory:

I. System (2.1) always has two exponential solutions

$$
m_{s}(t)=e^{-\mu_{m} t}, \quad f_{s}(t)=p(t) \equiv 0,
$$

and

$$
f_{s}(t)=e^{-\mu_{f} t}, \quad m_{s}(t)=p(t) \equiv 0 .
$$

II. If $\phi_{m}$ and $\phi_{f}$ denote the partial derivatives of $\phi$ with respect to the first and the second variable of $\phi$ respectively and if

$$
\mu_{m}<\mu_{f}-\frac{\beta_{f} \phi_{f}(1,0)}{\mu_{f}+\sigma+\phi_{f}(1,0)},
$$

then there is no strictly positive exponential solution, and the exponential solution in $I_{(a)}$ is trajectorally stable (see Hadeler and collaborators), while the solution expressed in $I_{(b)}$ is trajectorally unstable.

III. If

$$
\mu_{f}<\mu_{m}-\frac{\beta_{m} \phi_{m}(0,1)}{\mu_{m}+\sigma+\phi_{m}(0,1)}
$$

then there is no strictly positive exponential solution. The exponential solution $I_{(b)}$ is stable while the exponential solution in $I_{(a)}$ is unstable.

IV. If

$$
\mu_{m}>\mu_{f}-\frac{\beta_{f} \phi_{f}(1,0)}{\mu_{f}+\sigma+\phi_{f}(1,0)}
$$

and

$$
\mu_{f}>\mu_{m}-\frac{\beta_{m} \phi_{m}(0,1)}{\mu_{m}+\sigma+\phi_{m}(0,1)}
$$

then (2.1) has a unique strictly positive exponential solution, which is stable, while the solutions in $I_{(a)}$ and $I_{(b)}$ are both unstable.

\section{The Logistic Pair-Formation Model}

Because most of the demographic studies used models that exhibited exponential solutions (Malthus and Leslie), most of the work on demographic pairing models focussed 
on homogeneous systems. It is obviously important to study models that also incorporate nonlinear birth and separation processes. This article formulates the simplest nonlinear extension of the Dietz/Hadeler and Kendall/Keyfitz model that incorporates nonlinear birth and separation processes. Let $T=m_{s}+f_{s}+2 p$ (the total population), and assume that the birth rate $\beta=\beta(T)$ and the separation rate $\sigma=\sigma(T)$ depend on the total population. Furthermore, if we let $0<\gamma<1$ and $1-\gamma$ represent the proportion of the male and female birth rates respectively, then model (2.1) becomes

$$
\begin{aligned}
\dot{m}_{s} & =-\mu_{m} m_{s}+\left[\gamma \beta(T)+\mu_{f}+\sigma(T)\right] p-\phi\left(m_{s}, f_{s}\right) \\
\dot{f_{s}} & =-\mu_{f} f_{s}+\left[(1-\gamma) \beta(T)+\mu_{m}+\sigma(T)\right] p-\phi\left(m_{s}, f_{s}\right) \\
\dot{p} & =-\left[\mu_{m}+\mu_{f}+\sigma(T)\right] p+\phi\left(m_{s}, f_{s}\right),
\end{aligned}
$$

where we assume that $\beta$ and $\sigma$ satisfy the following properties:

$$
\begin{gathered}
\frac{d \beta(T)}{d T}<0, \quad \lim _{T \rightarrow \infty} \beta(T)=0 . \\
\frac{d \sigma(T)}{d T} \geq 0 .
\end{gathered}
$$

To simplify the analysis we introduce the following change of variables:

$$
\begin{gathered}
m=m_{s}+p, \\
f=f_{s}+p .
\end{gathered}
$$

The system (3.1) becomes

$$
\begin{aligned}
\dot{m} & =-\mu_{m} m+\gamma \beta(T) p \\
\dot{f} & =-\mu_{f} f+(1-\gamma) \beta(T) p \\
\dot{p} & =-\left[\mu_{m}+\mu_{f}+\sigma(T)\right] p+\phi(m-p, f-p),
\end{aligned}
$$

where $T=m+f$. With the assumptions (H1) and (H2), the system (3.2) can be considered as the generalization of the logistic equation. This generalization follows the dynamics of paired rather than single individuals. Since by definition we have $m \geq p$ and $f \geq p$, then we can focus only on solutions which belong to the set $\Omega$ defined as

$$
\Omega:=\left\{(m, f, p) \in \mathbb{R}_{+}^{3} ; \mathrm{p} \leq \mathrm{m}, \mathrm{p} \leq \mathrm{f}\right\} .
$$

It is not difficult to verify that $\Omega$ is positively invariant under the flows generated by the system (3.2). We now state our main results for the dynamics of System (3.2). The detailed mathematical analysis is provided in the next section. 
Theorem 3.1 If either

$$
\min \left\{\frac{\gamma \beta(0)}{\mu_{m}}, \frac{(1-\gamma) \beta(0)}{\mu_{f}}\right\} \leq 1
$$

or

$$
\min \left\{\frac{\gamma \beta(0)}{\mu_{m}}, \frac{(1-\gamma) \beta(0)}{\mu_{f}}\right\}>1
$$

and

$$
\mu_{m}+\mu_{f}+\sigma(0) \geq \phi\left(\frac{\gamma \beta(0)}{\mu_{m}}-1, \frac{(1-\gamma) \beta(0)}{\mu_{m}}-1\right)
$$

then for any given initial condition $z=\left(m_{0}, f_{0}, p_{0}\right) \in \Omega$

$$
\lim _{t \rightarrow \infty} m(t, z)=\lim _{t \rightarrow \infty} f(t, z)=\lim _{t \rightarrow \infty} p(t, z)=0 .
$$

Condition (3.3) implies that either the male growth rate or the female growth rate is negative, that is, either the total male population or the total female population vanishes and, consequently, so does the total paired population. Under the Condition (3.4) it is not obvious that the population goes to zero because both maximum male and female reproductive numbers, $\frac{\gamma \beta(0)}{\mu_{m}}$ and $\frac{(1-\gamma) \beta(0)}{\mu_{f}}$, exceed one. However, the second condition of (3.4) implies roughly that the growth rate for the paired individuals is negative and hence the rate of reproduction of the male and female populations is reduced.

Result 3.2 For system (3.2), if

$$
\begin{gathered}
\min \left\{\frac{\gamma \beta(0)}{\mu_{m}}, \frac{(1-\gamma) \beta(0)}{\mu_{f}}\right\}>1 \\
\mu_{m}+\mu_{f}+\sigma(0)<\phi\left(\frac{\gamma \beta(0)}{\mu_{m}}-1, \frac{(1-\gamma) \beta(0)}{\mu_{m}}-1\right) .
\end{gathered}
$$

then the zero solution is unstable and there exists a unique positive equilibrium $\left(m^{*}, f^{*}, p^{*}\right)$. Furthermore, the unique positive equilibrium is locally asymptotically stable.

If the total population $T$ is small, then condition (3.5) implies that the average growth rates of male, female and paired populations are positive (not completely accurate, see the proof of Lemma 3.2 in Section 4). Hence the total population persists, and so we expect the existence of a positive steady state.

\section{Mathematical Analysis of the Logistic Pair-Formation Model}


In this section we shall give the proofs of Result 3.1 and Result 3.2.

Proof of Result 3.1 We assume that condition (3.3) is satisfied, and without loss of generality we assume that

$$
\frac{\gamma \beta(0)}{\mu_{m}} \leq 1
$$

It follows that

$$
\begin{aligned}
\dot{m}(t) & =-\mu_{m}\left(1-\frac{\gamma \beta(T)}{\mu_{m}}\right) m(t)-\gamma \beta(T)[m(t)-p(t)] \\
& \leq-\mu_{m}\left(1-\frac{\gamma \beta(0)}{\mu_{m}}\right) m(t)-\gamma \beta(T)[m(t)-p(t)] .
\end{aligned}
$$

Since $m(t)-p(t) \geq 0$, then (4.1) implies that $m(t)$ is bounded and

$$
\lim _{t \rightarrow \infty}[m(t)-p(t)]=0 \text {. }
$$

Therefore, $p(t)$ and $f(t)$ are bounded and consequently

$$
\lim _{t \rightarrow \infty} \phi(m(t)-p(t), f(t)-p(t))=0 .
$$

¿From (4.2) and the last equation of (3.2) we have that

$$
\lim _{t \rightarrow \infty} p(t)=0 \text {. }
$$

Hence

$$
\lim _{t \rightarrow \infty} m(t)=\lim _{t \rightarrow \infty} f(t)=0 .
$$

We now assume that condition (3.4) is satisfied and that for any $z \in \Omega$ we let

$$
\eta(t)=\max \left\{\frac{\mu_{m} m(t)}{\gamma \beta(0)}, \frac{\mu_{f} f(t)}{(1-\gamma) \beta(0)}, p(t)\right\} \quad t \geq 0,
$$

where $m(t)=m(t, z), f(t)=f(t, z), p(t)=p(t, z)$. In fact, we have that

$$
\lim _{t \rightarrow \infty} \eta(t)=0
$$

To establish this result, first we show that

$$
\dot{\eta}(t)<0 \quad \text { whenever } \quad \eta(t)>0 .
$$

$$
\begin{aligned}
& \text { If } \eta(t)=\frac{\mu_{m} m(t)}{\gamma \beta(0)}>0 \text { and } T(t)=m(t)+f(t) \text { then } \\
& \dot{\eta}(t)=\frac{d}{d t}\left(\frac{\mu_{m} m(t)}{\gamma \beta(0)}\right) \\
& =\frac{\mu_{m}}{\gamma \beta(0)}\left[-\mu_{m} m(t)+\gamma \beta(T(t)) p(t)\right] \\
& <\frac{\mu_{m}}{\gamma \beta(0)}\left[-\mu_{m} m(t)+\gamma \beta(0) p(t)\right] \\
& =\mu_{m}\left[-\frac{\mu_{m} m(t)}{\gamma \beta(0)}+p(t)\right] \\
& \leq 0 \text {. }
\end{aligned}
$$


Using a similar argument it is easily shown that if

$$
\eta(t)=\frac{\mu_{f} f(t)}{(1-\gamma) \beta(0)}>0
$$

then

$$
\dot{\eta}(t)<0 \text {. }
$$

Furthermore if

$$
\eta(t)=p(t)>0
$$

then

$$
\frac{m(t)}{p(t)} \leq \frac{\gamma \beta(0)}{\mu_{m}}, \quad \frac{f(t)}{p(t)} \leq \frac{(1-\gamma) \beta(0)}{\mu_{f}}
$$

and

$$
\phi\left(\frac{m(t)}{p(t)}-1, \frac{f(t)}{p(t)}-1\right) \leq \phi\left(\frac{\gamma \beta(0)}{\mu_{m}}-1, \frac{(1-\gamma) \beta(0)}{\mu_{f}}-1\right) .
$$

Finally,

$$
\begin{aligned}
\dot{\eta}(t) & =\frac{d p(t)}{d t} \\
& =-\left[\mu_{m}+\mu_{f}+\sigma(T(t))\right] p(t)+\phi(m(t)-p(t), f(t)-p(t)) \\
& <-\left[\mu_{m}+\mu_{f}+\sigma(0)\right] p(t)+\phi(m(t)-p(t), f(t)-p(t)) \\
& =-p(t)\left[\mu_{m}+\mu_{f}+\sigma(0)-\phi\left(\frac{m(t)}{p(t)}-1, \frac{f(t)}{p(t)}-1\right)\right] \\
& \leq-p(t)\left[\mu_{m}+\mu_{f}+\sigma(0)-\phi\left(\frac{\gamma \beta(0)}{\mu_{m}}-1, \frac{(1-\gamma) \beta(0)}{\mu_{m}}-1\right)\right] \\
& \leq 0 .
\end{aligned}
$$

Thus we establish condition (4.4) and condition (4.3) follows from (4.1). Therefore

$$
\lim _{t \rightarrow \infty} m(t)=\lim _{t \rightarrow \infty} f(t)=\lim _{t \rightarrow \infty} p(t)=0 .
$$

The proof of Result (4.3) is completed.

To prove Result 3.2 we need following two lemmas:

Lemma 4.1 If

$$
\frac{\gamma \beta(0)}{\mu_{m}}>1, \quad \frac{(1-\gamma \beta(0))}{\mu_{m}}>1,
$$

and

$$
\mu_{m}+\mu_{f}+\sigma(0)<\phi\left(\frac{\gamma \beta(0)}{\mu_{m}}-1, \frac{(1-\gamma) \beta(0)}{\mu_{f}}-1\right)
$$


then the zero solution of (3.2) is unstable. Moreover, (3.2) has a unique positive equilibrium $\left(m^{*}, f^{*}, p^{*}\right)$.

Proof. We first prove that the zero solution is unstable. We are not able to do this in the customary way, that is, by linearizing the system around the origin because in general the homogeneous function $\phi$ is not differentiable at the origin. However, because of the assumptions of this lemma we can choose $T_{0}>0$ such that

$$
\mu_{m}+\mu_{f}+\sigma\left(T_{0}\right)<\phi\left(\frac{\gamma \beta\left(T_{0}\right)}{\mu_{m}}-1, \frac{(1-\gamma) \beta\left(T_{0}\right)}{\mu_{f}}-1\right) .
$$

In addition, if $z=\left(m_{0}, f_{0}, p_{0}\right) \in \Omega$ with $m_{0}>0, f_{0}>0, p_{0}>0$ then it is not difficult to see that

$$
m(t, z)>0, \quad f(t, z)>0, \quad p(t, z)>0, \quad \text { for } \text { all } t>0 .
$$

Furthermore, if $m_{0}+f_{0}<T_{0}$ and if we define

$$
\xi(t)=\min \left\{\frac{\mu_{m} m(t, z)}{\gamma \beta(0)}, \frac{\mu_{f} f(t, z)}{(1-\gamma) \beta(0)}, p(t, z)\right\}, \quad t \geq 0
$$

then using arguments used in the proof of Result 3.1 it can be shown that $\dot{\xi}(t)>0$ as long as $T(t)=m(t, z)+f(t, z)<T_{0}$. Therefore $T(t)$ eventually reaches $T_{0}$ and the zero solution is unstable.

The non-zero equilibria of the system (3.2) is determined by the solutions of the following nonlinear algebraic system of equations:

$$
\begin{aligned}
m & =\frac{\gamma \beta(T) p}{\mu_{m}} \\
f & =\frac{(1-\gamma) \beta(T) p}{\mu_{f}} \\
\mu_{m}+\mu_{f}+\sigma(T)-\phi\left(\frac{m}{p}-1, \frac{f}{p}-1\right) & =0 .
\end{aligned}
$$

Substitution of the first two equations into the last one leads to the following equation for $T$ :

$$
G(T):=\mu_{m}+\mu_{f}+\sigma(T)-\phi\left(\frac{\gamma \beta(T)}{\mu_{m}}-1, \frac{(1-\gamma) \beta(T)}{\mu_{f}}-1\right)=0 .
$$

Since by assumption we have

$$
G(0)=\mu_{m}+\mu_{f}+\sigma(0)-\phi\left(\frac{\gamma \beta(0)}{\mu_{m}}-1, \frac{(1-\gamma) \beta(0)}{\mu_{f}}-1\right)<0,
$$

then hypothesis (H1) implies the existence of constants $T_{m}>0$ and $T_{f}>0$ such that

$$
\frac{\gamma \beta\left(T_{m}\right)}{\mu_{m}}=\frac{(1-\gamma) \beta\left(T_{f}\right)}{\mu_{f}}=1
$$


If we let $\bar{T}=\min \left\{T_{m}, T_{f}\right\}$, then

$$
\phi\left(\frac{\gamma \beta(\bar{T})}{\mu_{m}}-1, \frac{(1-\gamma) \beta(\bar{T})}{\mu_{f}}-1\right)=0 .
$$

and consequently

$$
G(\bar{T})=\mu_{m}+\mu_{f}+\sigma(\bar{T})>0 .
$$

The fact that $G(T)$ is strictly monotone increasing, in combination with conditions (4.6) and (4.7), implies that $G(T)=0$ has a unique positive solution $T^{*}$. From the first two equations in (4.5) we have that

$$
p^{*}=T^{*} /\left(\frac{\gamma \beta\left(T^{*}\right)}{\mu_{m}}+\frac{(1-\gamma) \beta\left(T^{*}\right)}{\mu_{f}}\right)
$$

and

$$
m^{*}=\frac{\gamma \beta\left(T^{*}\right) p^{*}}{\mu_{m}}, \quad f^{*}=\frac{(1-\gamma) \beta\left(T^{*}\right) p^{*}}{\mu_{f}} .
$$

This concludes the proof of Lemma 4.1.

Lemma 4.2 The positive equilibrium is locally asymptotically stable.

Proof. An easy calculation shows that the linearization of (3.2) at the positive equilib$\operatorname{rium}\left(m^{*}, f^{*}, m^{*}\right)$ is

$$
\frac{a}{d t}\left[\begin{array}{c}
m \\
f \\
p
\end{array}\right]=A\left[\begin{array}{c}
m \\
f \\
p
\end{array}\right]
$$

where

$$
A=\left[\begin{array}{ccc}
-\mu_{m}+\gamma \dot{\beta}\left(T^{*}\right) p^{*} & \gamma \dot{\beta}\left(T^{*}\right) p^{*} & \gamma \beta\left(T^{*}\right) \\
(1-\gamma) \dot{\beta}\left(T^{*}\right) p^{*} & -\mu_{f}+(1-\gamma) \dot{\beta}\left(T^{*}\right) p^{*} & (1-\gamma) \beta\left(T^{*}\right) \\
\phi_{u}-\dot{\sigma}\left(T^{*}\right) p^{*} & \phi_{v}-\dot{\sigma}\left(T^{*}\right) p^{*} & -\mu_{m}-\mu_{f}-\sigma\left(T^{*}\right)-\phi_{u}-\phi_{v}
\end{array}\right]
$$

where $T^{*}=m^{*}+f^{*}$, and where

$$
\phi_{u}=\left.\frac{\partial}{\partial u} \phi(u, v)\right|_{(u, v)=\left(m^{*}-p^{*}, f^{*}-p^{*}\right)}, \quad \phi_{v}=\left.\frac{\partial}{\partial v} \phi(u, v)\right|_{(u, v)=\left(m^{*}-p^{*}, f^{*}-p^{*}\right)} .
$$

Using

$$
\phi\left(m^{*}-p^{*}, f^{*}-p^{*}\right)=\left(\mu_{m}+\mu_{f}+\sigma\left(T^{*}\right)\right) p^{*}
$$

and since for $\alpha>0$,

$$
\phi\left(\alpha\left(m^{*}-p^{*}\right), \alpha\left(f^{*}-p^{*}\right)\right)=\alpha \phi\left(m^{*}-p^{*}, f^{*}-p^{*}\right)=\alpha\left(\mu_{m}+\mu_{f}+\sigma\left(T^{*}\right)\right) p^{*}
$$


due to the homogeneity of $\phi$, then we see that

$$
\phi_{u}\left(m^{*}-p^{*}\right)+\phi_{v}\left(f^{*}-p^{*}\right)=\left(\mu_{m}+\mu_{f}+\sigma\left(T^{*}\right)\right) p^{*} .
$$

Equivalently

$$
\phi_{u} \frac{m^{*}}{p^{*}}+\phi_{v} \frac{f^{*}}{p^{*}}=\phi_{u}+\phi_{v}+\mu_{m}+\mu_{f}+\sigma\left(T^{*}\right) .
$$

¿From (4.8) and the equalities

$$
\mu_{m} \frac{m^{*}}{p^{*}}=\gamma \beta\left(T^{*}\right), \quad \mu_{f} \frac{f^{*}}{p^{*}}=(1-\gamma) \beta\left(T^{*}\right),
$$

we deduce that the characteristic polymonial $\operatorname{det}(\lambda I-A)$ is of the form

$$
\operatorname{det}(\lambda I-A)=\lambda^{3}+a_{1} \lambda^{2}+a_{2} \lambda+a_{3},
$$

with

$$
\begin{aligned}
a_{1}= & \mu_{m}+\mu_{f}-\dot{\beta}\left(T^{*}\right) p^{*}+\frac{1}{p^{*}}\left(\phi_{u} m^{*}+\phi_{v} f^{*}\right), \\
a_{2}= & \mu_{m} \mu_{f}+\beta\left(T^{*}\right) p^{*} \dot{\sigma}\left(T^{*}\right)+\frac{1}{p^{*}}\left(\mu_{f} m^{*} \phi_{u}+\mu_{m} f^{*} \phi_{v}\right) \\
& -\dot{\beta}\left(T^{*}\right)\left(\phi_{u} m^{*}+\phi_{v} f^{*}\right)-\left(\mu_{m}(1-\gamma)+\mu_{f} \gamma\right) \dot{\beta}\left(T^{*}\right) p^{*}, \\
a_{3}= & \dot{\sigma}\left(T^{*}\right) \beta\left(T^{*}\right) p^{*}\left(\mu_{m}(1-\gamma)+\mu_{f} \gamma\right)-\dot{\beta}\left(\mu_{m}(1-\gamma) f^{*} \phi_{v}+\mu_{f} \gamma m^{*} \phi_{u}\right) \\
& -\gamma(1-\gamma) \beta\left(T^{*}\right) \dot{\beta}\left(T^{*}\right) p^{*}\left(\phi_{u}+\phi_{v}\right) .
\end{aligned}
$$

¿From formula (4.9), (H1), and (H2) it follows that

$$
a_{1}>0, \quad a_{2}>0, \quad a_{3}>0,
$$

and

$$
\begin{aligned}
a_{1} a_{2}> & \dot{\sigma}\left(T^{*}\right) \beta\left(T^{*}\right) p^{*}\left(\mu_{m}+\mu_{f}\right) \\
& -\dot{\beta}\left(T^{*}\right)\left(\mu_{m}(1-\gamma) m^{*} \phi_{u}+\mu_{f}(1-\gamma) f^{*} \phi_{v}+\mu_{m} \gamma m^{*} \phi_{u}+\mu_{f} \gamma f^{*} \phi_{v}\right) \\
> & \left.\dot{\sigma}\left(T^{*}\right) \beta\left(T^{*}\right) p^{*}\left(\mu_{m}(1-\gamma)+\mu_{f} \gamma\right)-\dot{\beta}\left(T^{*}\right)\left(\mu_{f} \gamma\right) m^{*} \phi_{u}+\mu_{m}(1-\gamma) f^{*} \phi_{v}\right) \\
& -\dot{\beta}\left(T^{*}\right)\left(\mu_{m}(1-\gamma) m^{*} \phi_{u}+\mu_{f} \gamma f^{*} \phi_{v}\right) \\
= & \left.\dot{\sigma}\left(T^{*}\right) \beta\left(T^{*}\right) p^{*}\left(\mu_{m}(1-\gamma)+\mu_{f} \gamma\right)-\dot{\beta}\left(T^{*}\right)\left(\mu_{f} \gamma\right) m^{*} \phi_{u}+\mu_{m}(1-\gamma) f^{*} \phi_{v}\right) \\
& -\dot{\beta}\left(T^{*}\right) \beta\left(T^{*}\right) p^{*} \gamma(1-\gamma)\left(\phi_{u}+\phi_{v}\right) \\
= & a_{3} .
\end{aligned}
$$

By applying the Routh Hurwitz criteria we conclude that all zeros of the characteristic polynomial $\operatorname{det}(\lambda I-A)$ have negative real parts. Hence the positive equilibrium is locally asymptotically stable. 
Proof of Result 3.2 Result 3.2 is a direct consequence of Lemmas 4.1 and 4.2.

Remark We have only confirmed the local stability of the positive equilibrium. We do not know whether or not the positive equilibrium is globally stable but our conjecture is that it is.

\section{Conclusions}

Demography and population dynamics (life history theory) have been developed, at least in spirit, along the lines of single sex-models that have as their basis the Malthus and logistic models. The introduction of age-structured models followed a similar pattern through the introduction of Malthusian models such as the Leslie and MacKendrick/Lotka (also the Von Foerster) models (see Hoppensteadt 1974) and nonlinear logistic-type models such as the Gurtin/MacCamy model. In this article we have revisited a Malthusian pairformation model and have introduced a logistic-type pair-formation model. Both models have to include, in addition to the standard birth-death process, pair-formation and dissolution processes. If the birth-death process is linear then we obtain the Malthusian pair-formation model of Kendall/Keifitz and Dietz/Hadeler which supports exponential solutions despite the inclusion of a nonlinear pair-formation process. Furthermore, this model supports a unique (trajectorally) stable exponential solution. If the birth/death process and the dissolution rate are nonlinear then we have a logistic-type pair-formation model that can support a unique nontrivial locally-stable bounded solution (we suspect that it is globally stable). Despite the fact that we have now replaced single equations by systems of equations, Malthusian and logistic two-sex models exhibit the same qualitative dynamics as their associated single-sex models. If further structure is added, such as age-structure, then several mathematical extensions are possible.

\section{Acknowledgements}

This research was partially supported by NSF grant DEB-925370 to Carlos CastilloChavez and by the U.S. Army Research Office through the Mathematical Science Institute of Cornell University (contract DAAL03-91-C-0027). This article was completed while Carlos Castillo-Chavez was a Fellow at the Department of Ecology and Evolutionary Biology. 


\section{REFERENCES}

[1] Castillo-Chavez, C., Velasco-Hernàndez, J. X.\& Fridman, S. (1993). Modeling Contact Structures in Biology. In (S. A. Levin ed.), Frontiers of Theoretical Biology, Lecture Notes in Biomathematics 100 (in press). Berlin, Heidelberg, New York, London, Paris, Tokyo, Hong Kong, Barcelona, Budapest: Springer- Verlag.

[2] Castillo-Chavez, C. \& Busenberg, S. (1991), On the solution of two-sex problem. In: Proceedings of the International Conference on Differential Equations and Applications to Biology and Population Dynamics (S. Busenberg \& M. Martelli, eds.), Lecture Notes in Biomathematics, 80-98, Springer-Verlarg, Berlin-Heidelberg-New YorkLondon-Paris-Tokyo-Hong Kong, Barcelona, Budapest.

[3] Castillo-Chavez, C., W. Huang, \& J. Li (1993), Dynamics for a pair formation model of multiple groups, preprint.

[4] Dietz, K \& Hadeler, K.R.(1988), Epidemiological models for sexually transmitted diseases, J. Math. Biol. 26, 1-25.

[5] Fredrickson, A.G.(1971), A mathematical theory of age structure in sexual populations: Random mating and monogamous marriage models, Math. Biosci. 20, 117-143.

[6] Gurtin, ME. \& C.R. MacCAmy (1974), Non-Linear age dependent population dynamics, Archive for Rational Mechanic and Analysis, 54 (3), 281-300.

[7] Hoppensteadt, F. (1975), Mathematical Theories of Populations: Demographics, Genetics and Epidemics (SIAM Regional Conference Series in Applied Math. num 20, Filalfia.

[8] Leslie, PH.(1945), On the use for matrices in certain population mathematics, Biometrika, 33, 183-212.

[9] Kendall, D.G. (1949), Stochastic processes and population growth, Roy. Statist. Soc., Ser.B2:230-264.

[10] Keyfitz, N. (1949), The mathematics of sex and marriage, Proceedings of the Sixth Berkeley Symposium on Mathematical Statistics and Probability, Vol. IV: Biology and Health, 89-108.

[11] Malthus, TR.(1978), An Essay on the Principle of Population (1a Ed), J Johnson in St Paul's Churchyard, London.

[12] May, RM. (1974), Stability and Complexity in Model Ecosystems (2a Ed), Princeton Univ. Press, Princeton. 
[13] McFarland, D. (1972), Comparison of alternative marriage models. In (Greville, T. N. E., ed.), Population Dynamics, Academic Press, New York London: 89-106.

[14] Parlett, B. (1972), Can there be a marriage function? In (Greville, T. N. E., ed.), Population Dynamics, Academic Press, New York London: 107-135.

[15] Pollard, J.H. (1973), Mathematical models for the growth of human populations, Chapter 7: The two sex problem, Cambridge University Press.

[16] Schmitz, S.F, Busenberg, S. \& Castillo-Chavez, C. (1993), On the evolution of marriage functions: It takes two to tango, Biometrics Unit Technical Report BU-1210-M, Cornell University, Ithaca, New York.

[17] Verhulst, P.F. (1845), Recherches mathématiques sur la loi d'accroissement de la population, Mém Acad Roy, Brussels, 18 (1).

[18] Verhulst, P.F. (1847), Recherches mathématiques sur la loi d'accroissement de la population, Mém Acad Roy, Brussels, 20 (1).

[19] Waldstätter, R. (1989), Models for pair formation with applications to demography and epidemiology, Ph.D Dissertation. 